\title{
The Rolling Bearing Fault Feature Extraction Based on the LMD and Envelope Demodulation
}

\author{
Jun Ma, ${ }^{1}$ Jiande Wu, ${ }^{1,2}$ Yugang Fan, ${ }^{1,2}$ and Xiaodong Wang ${ }^{1,2}$ \\ ${ }^{1}$ Faculty of Information Engineering and Automation, Kunming University of Science and Technology, \\ Kunming 650500, China \\ ${ }^{2}$ Engineering Research Center for Mineral Pipeline Transportation YN, Kunming 650500, China
}

Correspondence should be addressed to Jiande Wu; wjiande@foxmail.com

Received 30 September 2014; Revised 1 January 2015; Accepted 5 January 2015

Academic Editor: Xinggang Yan

Copyright (C) 2015 Jun Ma et al. This is an open access article distributed under the Creative Commons Attribution License, which permits unrestricted use, distribution, and reproduction in any medium, provided the original work is properly cited.

\begin{abstract}
Since the working process of rolling bearings is a complex and nonstationary dynamic process, the common time and frequency characteristics of vibration signals are submerged in the noise. Thus, it is the key of fault diagnosis to extract the fault feature from vibration signal. Therefore, a fault feature extraction method for the rolling bearing based on the local mean decomposition (LMD) and envelope demodulation is proposed. Firstly, decompose the original vibration signal by LMD to get a series of production functions (PFs). Then dispose the envelope demodulation analysis on PF component. Finally, perform Fourier Transform on the demodulation signals and judge failure condition according to the dominant frequency of the spectrum. The results show that the proposed method can correctly extract the fault characteristics to diagnose faults.
\end{abstract}

\section{Introduction}

Rolling bearing is one of the most important and the easiest failure components of rotating machinery. The faults of bearing will affect the normal operation of equipment and even cause a series of serious accidents. So the fault diagnosis of rolling bearing is vitally important [1]. The fault diagnosis of bearing based on the vibration signal is always used. But the running process of rolling bearings is a complex and nonstationary dynamic process; the time domain and frequency domain characteristics of vibration signals are not obvious enough to diagnose the bearing fault [2]. The feature extraction of the vibration signal, therefore, plays a key role in the fault diagnosis.

In the process of fault feature extraction, the envelope demodulation analysis is one of the most commonly used methods, mainly including the Hilbert Transform (HT) and Teager energy operator (TEO) demodulation [3]. Envelope demodulation analysis is widely used in speech recognition, fault diagnosis, and other fields $[1,4-6]$. The demodulation methods mentioned above are based on the single amplitude modulation and frequency modulation (AM-FM) signal, but the fault vibration signal of rolling bearing is a multicomponent AM-FM modulation signal. Therefore, the signal decomposition is a must before analyzing and processing the signal with the HT and TEO demodulation. The decomposition methods frequently used are the wavelet or wavelet packet decomposition, empirical mode decomposition (EMD), ensemble empirical mode decomposition (EEMD), LMD, and other signal decomposition methods. The EMD, EEMD, and LMD are adaptive decomposition methods based on the characteristics of the signal. In order to filter the noise in the stator current signal produced by the broken rotor bar, the EMD is used in the literature [7], and the HT is used to extract the signal envelope to filter the noise. Finally, extract the fault characteristic frequency of the rotor broken bar and perform FFT on the envelope. In order to overcome the drawbacks of resonance frequency in the traditional envelope analysis, the method based on EMD and HT demodulation is put forward $[8,9]$. Locate the resonance frequency band to the concrete IMF component and realize the band filtering by the EMD and then select 
appropriate IMF component to conduct envelope analysis and judge the bearing working condition by its spectrum. Feng acknowledged that wind turbine gear vibration signals are also the multicomponent AM-FM modulation signal which are polluted by noise and put forward to the new analysis method combining EEMD with TEO for fault diagnosis. The experiments results show that the method can effectively diagnose the wind turbine gear fault [10].

Smith presented a new adaptive processing method for the nonstationary signal based on the EMD and EEMD research which is nominated LMD [11]. It can decompose any complex nonstationary signal into several PF components and ensure that the instantaneous frequency has physics meaning for each PF component. For comparing with EMD and EEMD, it overcame the negative frequency that cannot be interpreted with EMD. The boundary effect and the mode mixing have small impact on LMD [12]. So it is widely used in the field of fault diagnosis. The vibration signals of the main components (the rotor, the gear, and rolling bearing) in rotating machinery are multicomponent AM-FM modulation signals. In order to judge the bearing working condition, the instantaneous frequency of PFs decomposed is calculated by the introduced LMD and we compared the performance of the EMD with LMD's $[13,14]$. Aiming at the problem that the early fault diagnosis of the motor is restricted by the low speed of the vibration composition, Liu et al. introduced the LMD decomposition methods and decomposed the wind generator fault vibration signal by the LMD and calculated the instantaneous frequency of PFs component to diagnose the fault [15]. Deng and Zhao combined LMD with FFT and directly analyzed the FFT spectrum of the PFs and then selected appropriate PFs component according to the spectrum feature and restructured fault signal to describe the mechanical working condition [16].

In the fault diagnosis field of the rolling bearing, the research which combines LMD and modern digital signal processing receives more attention. Yet, according to the energy distribution of PFs obtained by LMD, the research about selecting appropriate PFs component for envelope demodulation analysis to diagnose faults and comparing the performance of demodulation method is few. Therefore, a method of fault feature extraction for rolling bearing, combining LMD with envelope demodulation, is presented. Firstly, decompose the vibration signal of the rolling bearing by the LMD. Then dispose the envelope demodulation analysis on PF including HT and TEO demodulation. Secondly, perform the Fourier Transform on the demodulation signals and make a judgment of rolling bearing failure condition by matching the dominant frequency components of the spectrum with fault characteristic frequency of rolling bearing. Finally, compare the final performance of TEO demodulation with HT's.

The paper is organized as follows. The LMD and envelope demodulation of the vibration signal are, respectively, introduced in Section 2. In Section 3, the fault feature extraction of the rolling bearing by the LMD and envelope demodulation is proposed. Section 4 demonstrates the experiment results analysis. The actual experimental analysis is presented in Section 5. Conclusions are obtained in Section 6.

\section{LMD and Envelope Demodulation of the Vibration Signal}

2.1. The LMD of Vibration Signal. The essence of the LMD method is to separate the pure frequency modulation signal and envelope signal from the original signal. A PF component with physical meaning instantaneous frequency is obtained by multiplying the pure frequency modulation signal with the envelope signal. All the PFs are obtained by iterative processing. The detailed decomposition process of the LMD can refer to the literature [12]. According to energy distribution of the PFs, the appropriate PF is selected for subsequent analysis of the envelope demodulation.

2.2. Envelope Demodulation of the Vibration Signal. The envelope demodulation method synthesizes the envelope detection and spectral analysis of the signal. The bearing fault can be identified by using the peak of the envelop spectrum. When the local damage or defects of the rolling bearing happen, the process of the load will produce mutational decaying shock pulse force and high frequency natural vibration of bearing. The high frequency natural vibration is regarded as a carrier of bearing vibration signal. Its amplitude will be subject to the modulation of pulse exciting force. Therefore, the final vibration signal of bearing is a complex amplitude modulation wave. Since the frequency of the modulation wave is corresponding to the passing frequency of the defects, it is possible to use envelope demodulation for fault diagnosis of the rolling bearing. In this dissertation, the HT and TEO demodulation are introduced to extract defect information from AM vibration signals of the rolling bearing.

2.2.1. HT Demodulation. The HT envelope demodulation principle is to rotate the test signal by 90 degrees and then combine it with the original signal to constitute an analytic signal [17]. The HT of a real signal is defined as follows:

$$
x_{h}(t)=H[x(t)]=\frac{1}{\pi} \int_{-\infty}^{+\infty} \frac{x(\tau)}{t-\tau} d \tau=x(t) \times \frac{1}{\pi t} .
$$

The analytic signal of $x(t)$ is $g(t)$ :

$$
g(t)=x(t)+j x_{h}(t) .
$$

The expression of amplitude $A(t)$ and phase $\varphi(t)$ of the analytic signal $g(t)$ are shown as follows:

$$
g(t)=A(t) e^{j \varphi(t)}
$$

where,

$$
\begin{aligned}
& A(t)=\sqrt{x^{2}(t)+x_{h}^{2}(t),} \\
& \varphi(t)=\tan ^{-1}\left[\frac{x_{h}(t)}{x(t)}\right] .
\end{aligned}
$$

Calculate envelope signal $A(\mathrm{PF})$ of $\mathrm{PF}$ components decomposed by the LMD based on (1), (2), and (4). The original signal is substituted for the $A(\mathrm{PF})$ containing the defect vibration component to dispose subsequently Fourier spectral analysis and extract the fault feature of vibration signal. 
2.2.2. TEO Demodulation. The TEO for the continuous signal $x(t)$ is defined as follows in the literature [18]:

$$
\varphi[x(t)]=[\dot{x}(t)]^{2}-x(t) \ddot{x}(t),
$$

where $\dot{x}(t)$ and $\ddot{x}(t)$, respectively, represent the first and second order differential of $x(t)$. The differential is substituted for the difference for the discrete signal $x(n)$. The TEO is defined as follows:

$$
\varphi[x(n)]=[x(n)]^{2}-x(n-1) x(n+1) .
$$

In (7), the TEO can be calculated for the source of signal energy at any time $n$ by using 3 samples. The $\varphi[\mathrm{PF}]$ of PFs can be calculated according to (7). The original signal is replaced by the $\varphi[\mathrm{PF}]$ containing the defect vibration component to dispose subsequently Fourier spectral analysis and extract the fault feature of vibration signal.

\section{The Fault Feature Extraction Process of the Rolling Bearing by LMD and Envelope Demodulation}

The fault vibration signal of rolling bearing is multicomponent AM-FM signals. A series of PF components which multiply an envelope signal with a pure FM signal are obtained by performing LMD on the original vibration signal. According to the energy distribution of PFs, the appropriate PF component which is energy concentrated can be selected to dispose subsequently envelope demodulation analysis, including HT and TEO demodulation, and get, respectively, amplitude envelope $A(\mathrm{PF})$ and $\varphi[\mathrm{PF}]$. Perform Fourier Transform on the demodulation signals $A(\mathrm{PF})$ and $\varphi[\mathrm{PF}]$ and make a judgment of rolling bearing working condition by matching the dominant frequency components of the spectrum with fault characteristic frequency of rolling bearing. Finally, compare the final performance of TEO demodulation with HT's.

The specific steps are as follows.

(1) The original vibration signal is decomposed by LMD. Then extract the appropriate PF component from all PFs to dispose subsequently analysis according to the energy distribution of PFs.

(2) The selected PF components are demodulated by HT and TEO to obtain the amplitude $A(\mathrm{PF})$ and phase $\varphi[\mathrm{PF}]$ respectively.

(3) The original signals are substituted for the $A(\mathrm{PF})$ and $\varphi[\mathrm{PF}]$ to obtain the Fourier spectrum through the Fourier Transform.

(4) Recognize the fault condition of the rolling bearing by matching the frequency spectrum with the fault characteristic frequency of theoretical calculation.

The detailed algorithm flowchart is shown in Figure 1.

\section{Results and Discussion}

In order to verify the effectiveness of the proposed method, the rolling bearing experimental data of Case Western

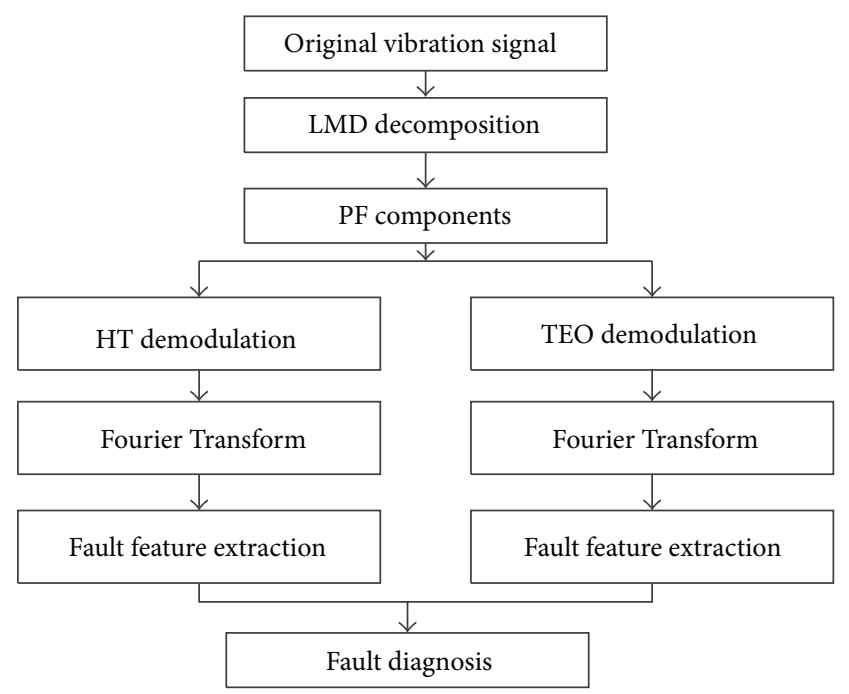

FIGURE 1: The detailed fault diagnosis processing of the rolling bearing.

TABLE 1: Fault characteristic frequency (unit: $\mathrm{Hz}$ ).

\begin{tabular}{lcc}
\hline Outer ring fault & Inner ring fault & Rolling element failure \\
\hline 107.3648 & 162.1852 & 141.1693 \\
\hline
\end{tabular}

Reserve University in electrical engineering laboratory is used as the test data for validation [19]. The detailed experiment parameters are as follows. The specification of the rolling bearing is 6205-2RSJEMSKF; the acceleration sensor is installed on the motor drive; the motor speed is $1797 \mathrm{rpm}$ (namely, the rotational frequency is $1797 / 60 \mathrm{~Hz}=$ $29.95 \mathrm{~Hz}$ ). The sampling frequency of the data acquisition system is $12 \mathrm{KHz}$. The experimental data length is 2048 . Respectively, select the normal signal of rolling bearing, the vibration signal of out ring fault, inner ring fault, and rolling bearing fault to do experimental analysis. The diameter of out ring fault, inner ring fault, and roller fault is 0.007 inches $(0.01778 \mathrm{~cm})$.

The bearing fault characteristic frequency is calculated as shown in Table 1, according to the above-mentioned bearing parameters and the theoretical formula of rolling bearing fault characteristic frequency in the literature [20].

4.1. Bearing Normal Vibration Signal Analysis. The results of time and frequency domain and LMD decomposition of rolling bearing normal vibration signal are shown in the Figure 2. Due to the influence of noise and interference, it has been unable to directly extract the bearing fundamental frequency characteristics from the time-frequency domain. Therefore, decompose the bearing normal vibration signal into several PFs by using the LMD algorithm and select the effective PF components according to the energy distribution of the PFs. The results are as shown in Figure 3.

As seen in Figure 3, the PF5 component in the total energy is relatively small and it is regarded as a pseudocomponent to be removed. Inversely, the PF1 component contains wealthy 

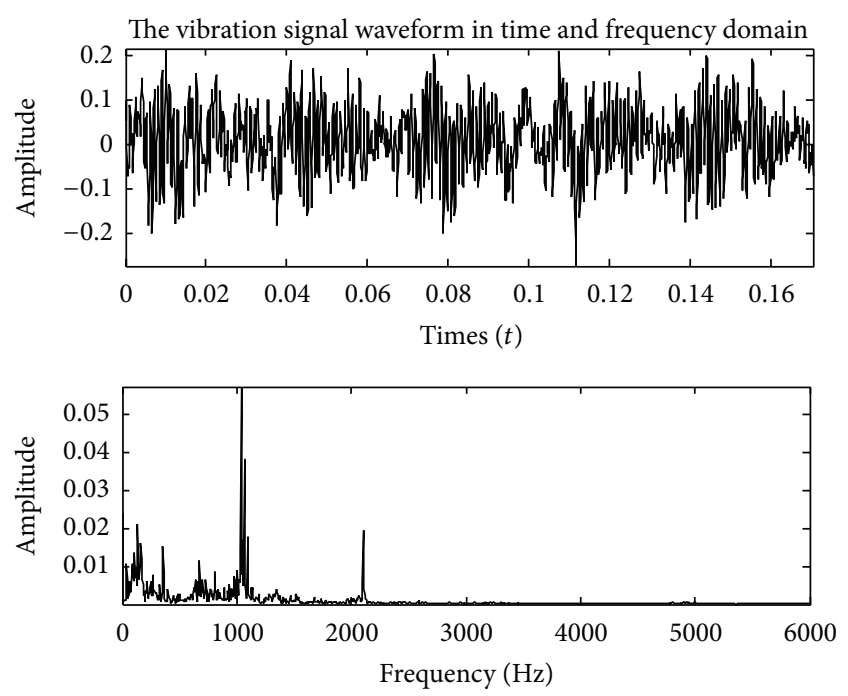

(a) The time domain and frequency domain waveform of normal vibration signal
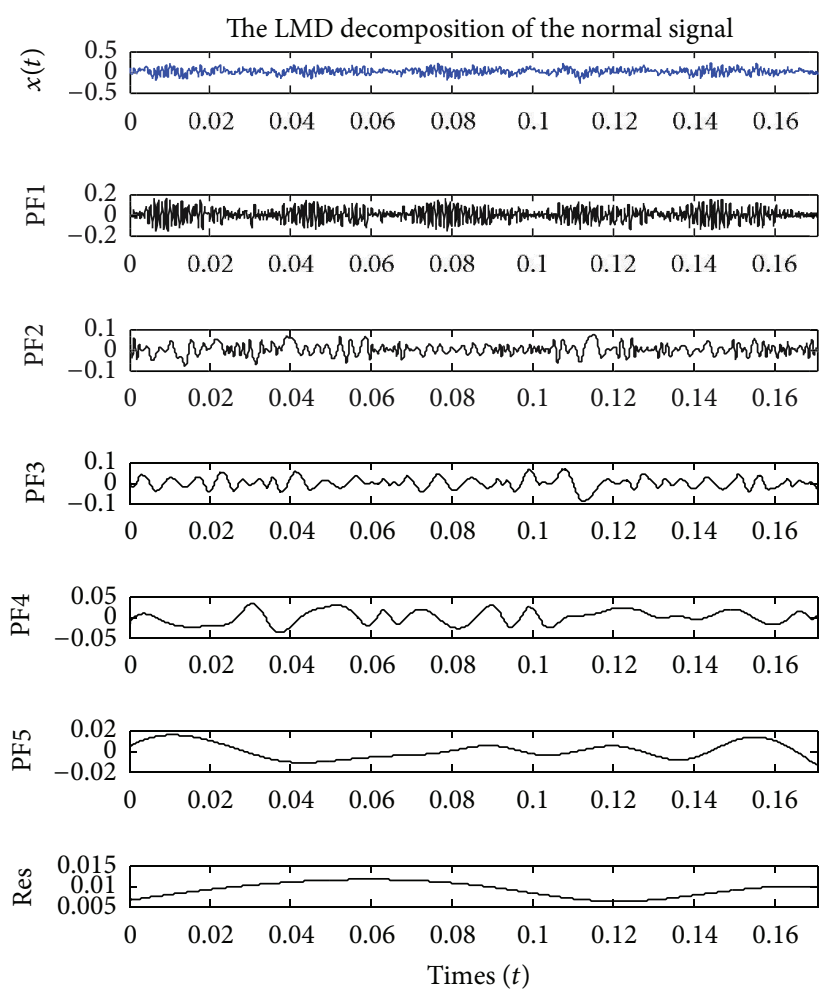

(b) The normal vibration signal LMD decomposing PF component

FIGURE 2: The time domain, frequency domain waveform and the LMD decomposition result of the normal vibration signal.

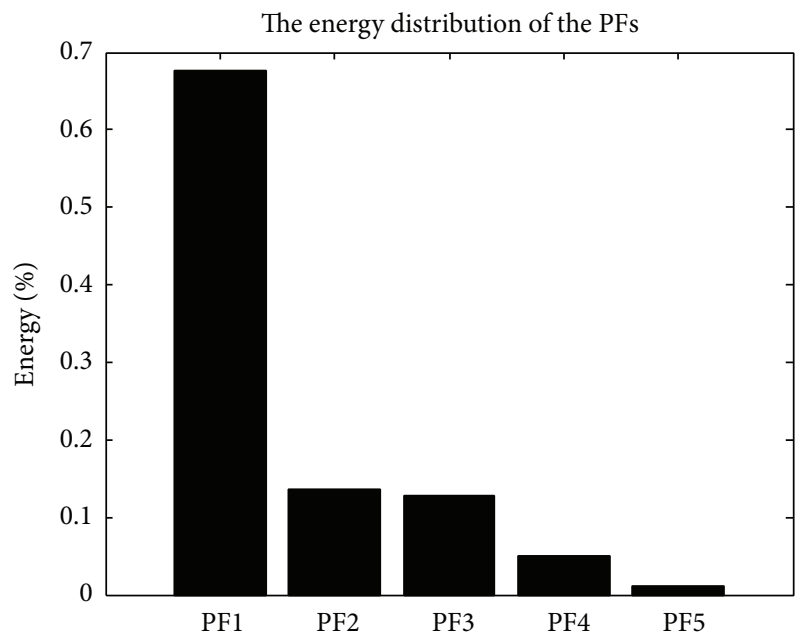

FIGURE 3: The normal bearing vibration signal PF component energy distribution.

information of original signals. So, select the PF1 as an effective component for subsequent envelope demodulation. The envelope demodulation result is shown in Figure 4.

As shown in Figure 4, the frequency with $29.3 \mathrm{~Hz}$ and its doubling frequency are very close to the rotation fundamental frequency and the frequency doubling of the bearing normal operation. Because of the influence of noise and parameter error, there has been small range of error between calculated and the measured values of the fundamental frequency. We, therefore, can judge that it is the rotation fundamental frequency of the bearing normal operation. At the same time, a peak at the frequency with $87.89 \mathrm{~Hz}$ has appeared, which is very close to three times of bearing fundamental frequency whose value is $89.85 \mathrm{~Hz}$. Furthermore, the experiment results show that the TEO envelope demodulation performance is better than HT's by comparing Figure 4(a) with Figure 4(b).

4.2. Inner Ring Fault Vibration Signal Analysis. The results of time-frequency domain and LMD decomposition of bearing inner ring fault vibration signal are shown in Figure 5. The $\mathrm{PF}$ component energy distribution is shown in Figure 6. In a similar way, select the PF1 as an effective component for subsequent envelope demodulation.

In Figure 7, the PF1 component has an obvious peak at the 158.2 Hz, which is relatively close to $162.1852 \mathrm{~Hz}$ of bearing inner ring fault frequency theoretical calculation value, as well as the double frequency with $316.4 \mathrm{~Hz}$. In addition, a peak at the frequency with $59.9 \mathrm{~Hz}$ has appeared in Figure 7, which is very close to two times of bearing fundamental frequency with $59.9 \mathrm{~Hz}$. We synthetically take the influence of inner parameter error and transmission path and so on into account. There are some errors between the theoretical calculation and measured values of inner fault characteristic frequency. Thus we can judge that the bearing exhibits inner 


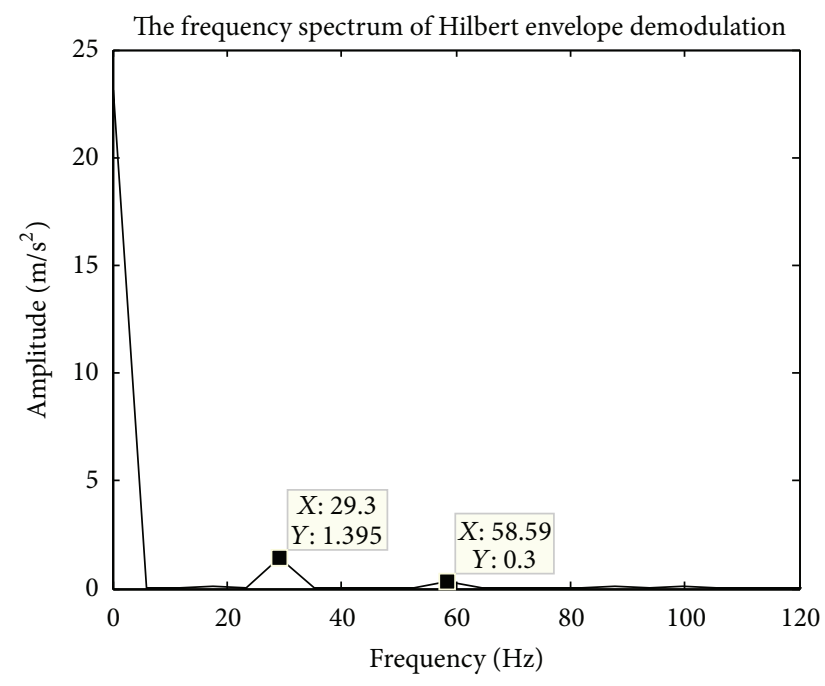

(a) Hilbert envelope demodulation spectrum

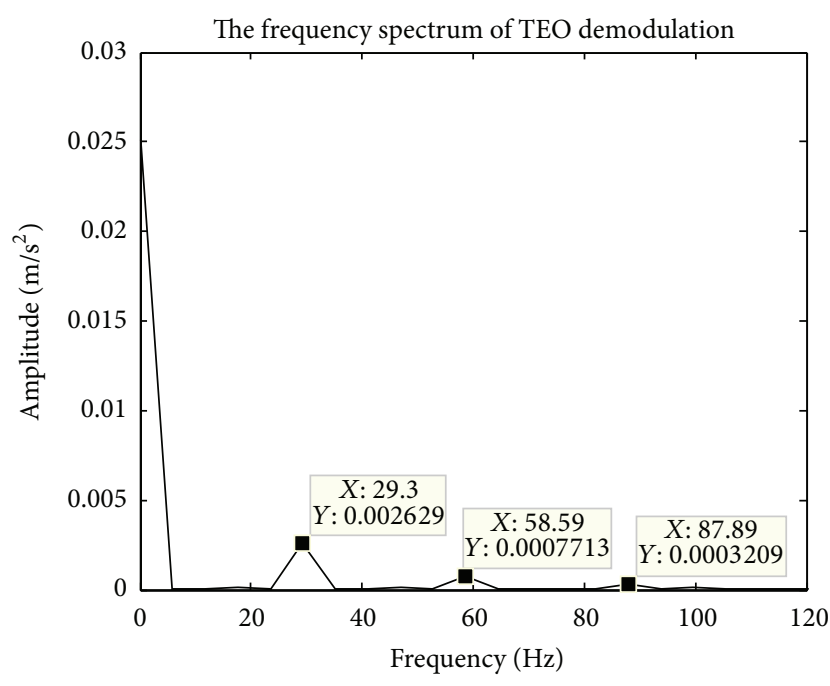

(b) TEO envelope demodulation spectrum

FIGURE 4: The Hilbert of normal rolling bearing vibration signal and TEO envelope demodulation spectrum.
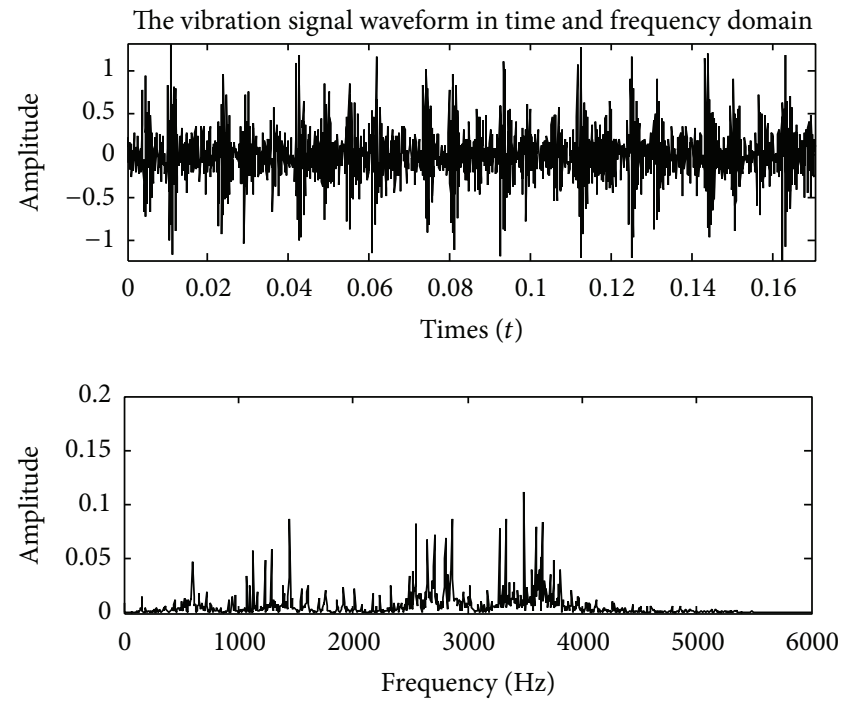

(a) Time domain, frequency domain waveform

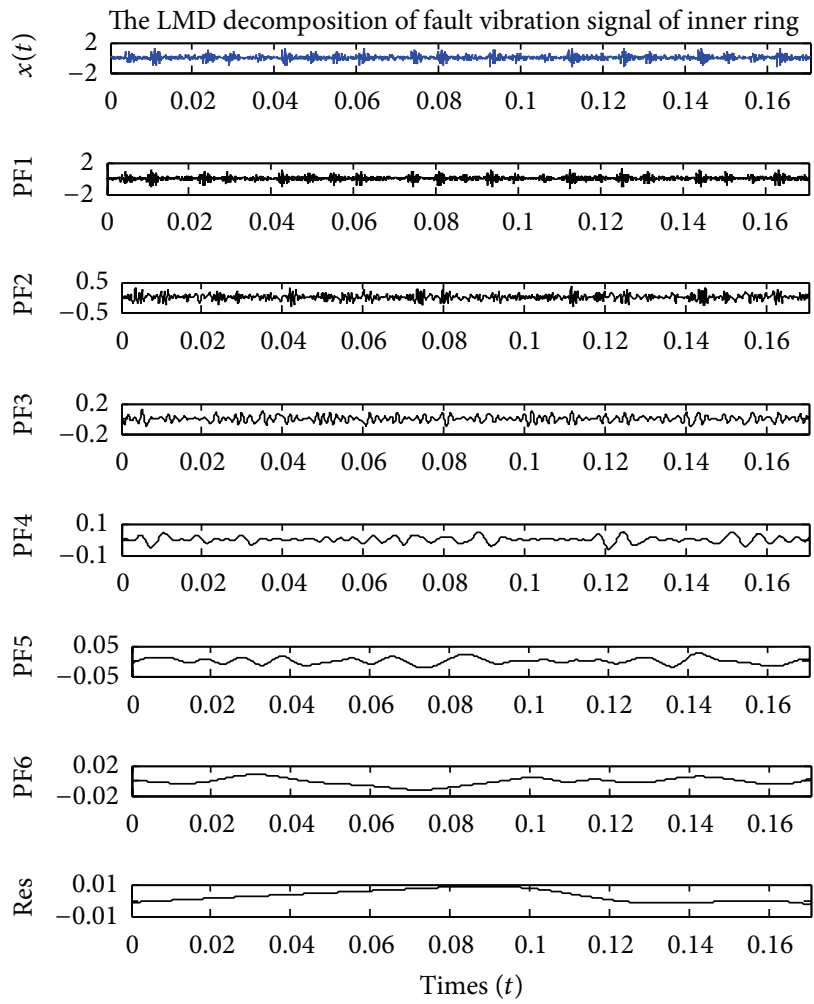

(b) PF component of inner ring fault vibration signal LMD decomposition

FIGURE 5: The time domain, frequency domain waveform, and the LMD decomposition result of the inner ring fault vibration signal.

ring fault at frequency whose value is $158.2 \mathrm{~Hz}$. Furthermore, the detected characteristic frequency peak and frequency doubling by using the TEO envelope demodulation are more obvious than HT's. It can help for improving the recognition precision and reliability.
4.3. Outer Ring Fault Vibration Signal Analysis. The results of time-frequency domain and LMD decomposition of rolling bearing outer ring fault vibration signal are shown in Figure 8. The energy distribution of PF component is shown in Figure 9. We can see the following. 


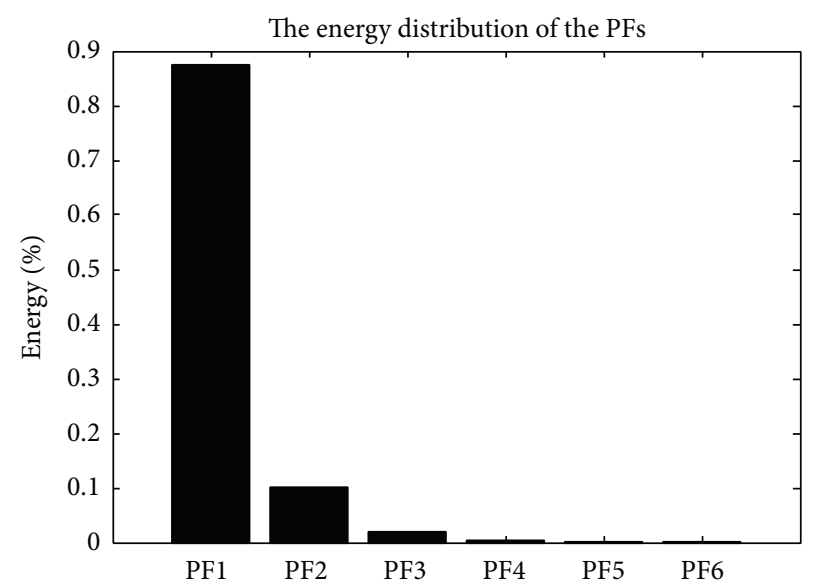

FIGURE 6: The energy distribution of inner ring vibration fault signal.

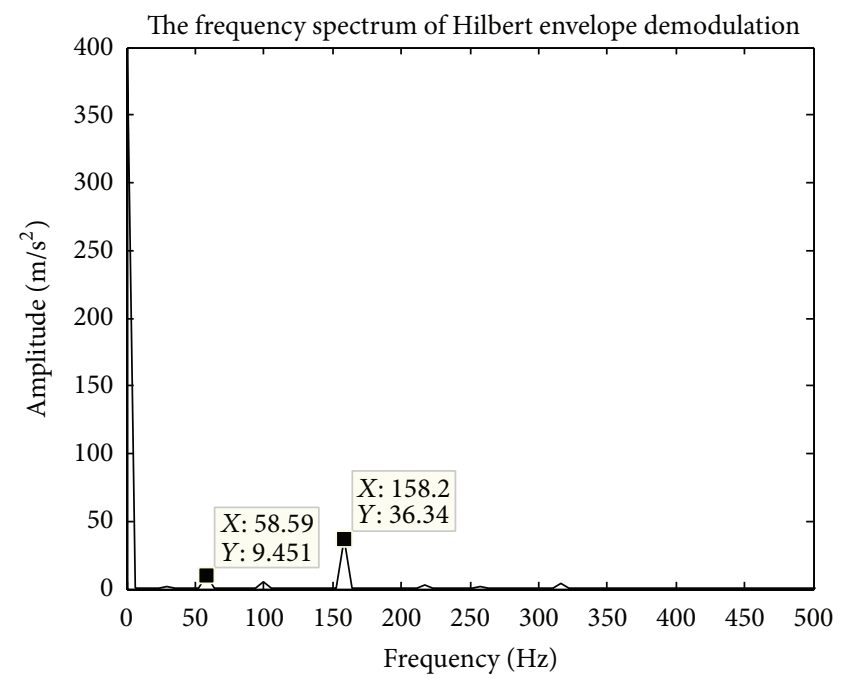

(a) Hilbert envelope spectrum

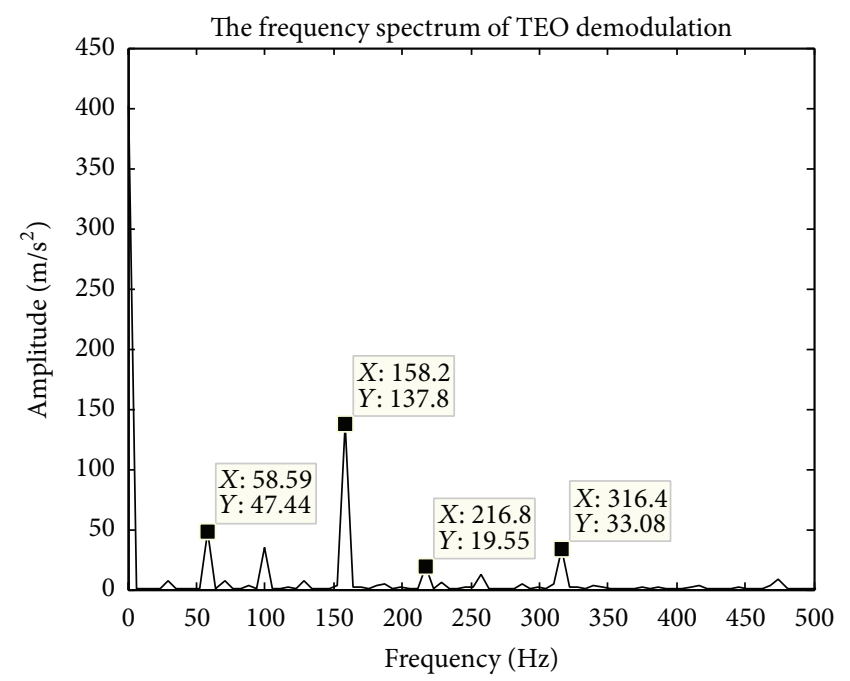

(b) TEO envelope spectrum

FIgURE 7: The Hilbert and TEO envelope spectrum of the rolling bearing inner ring fault vibration signals.

The energy percentage between PF3 and PF6 is small, which will be regarded as a pseudocomponent to be excluded. PF1 component contains wealthy information of original signal. So, the PF1 component is selected as an effective component for envelope demodulation. The envelope demodulation result is shown in Figure 10.

Figure 10 shows the characteristic frequency with $29.3 \mathrm{~Hz}$ which is similar to the bearing fundamental frequency with $29.95 \mathrm{~Hz}$ and outer ring fault characteristic frequency with $107.3648 \mathrm{~Hz}$ close to the frequency with $105.5 \mathrm{~Hz}$. There exists obvious doubling frequency characteristic. Because of the influence of inner parameter error and transmission path, there are certain errors between the theoretical calculation and measured values of rolling fault frequency. Thus we can judge that bearing exhibits outer ring fault at the frequency with $105.5 \mathrm{~Hz}$. At the same time, it is obvious that the effect of using TEO demodulation is better than Hilbert.
4.4. Roller Fault Vibration Signal Analysis. The results of time-frequency domain and the LMD decomposition of rolling bearing rolling fault vibration signals are shown in Figure 11. The energy distribution of PF component is shown in Figure 12. In Figure 12, the energy percentage between PF4 and PF6 is small, which can be regarded as a pseudocomponent to be excluded. The PF1 component is selected as an effective component for envelope demodulation. The demodulation analysis result is shown in Figure 13.

Figure 13 shows the characteristic frequency with $58.59 \mathrm{~Hz}$ which is similar to the bearing fundamental frequency of double frequency with $59.9 \mathrm{~Hz}$. At the same time, there is an obvious spectrum peak at the frequency with $140.6 \mathrm{~Hz}$. The experiment results show that there exists a small range of errors between the theoretical calculation and measured values of bearing fault frequency. Thus we can judge that the bearing xhibits rolling element fault 

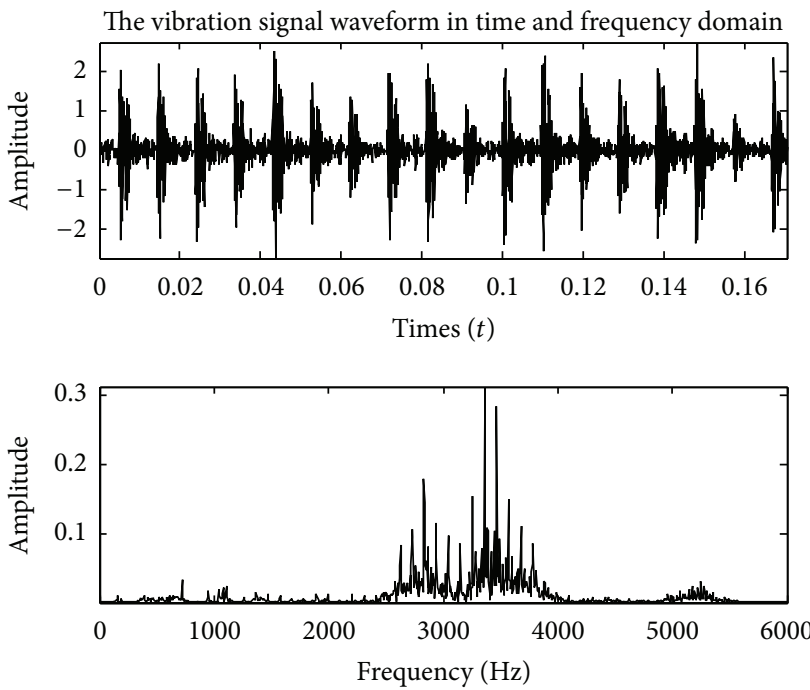

(a) Time domain and frequency domain waveform of outer ring fault vibration signal
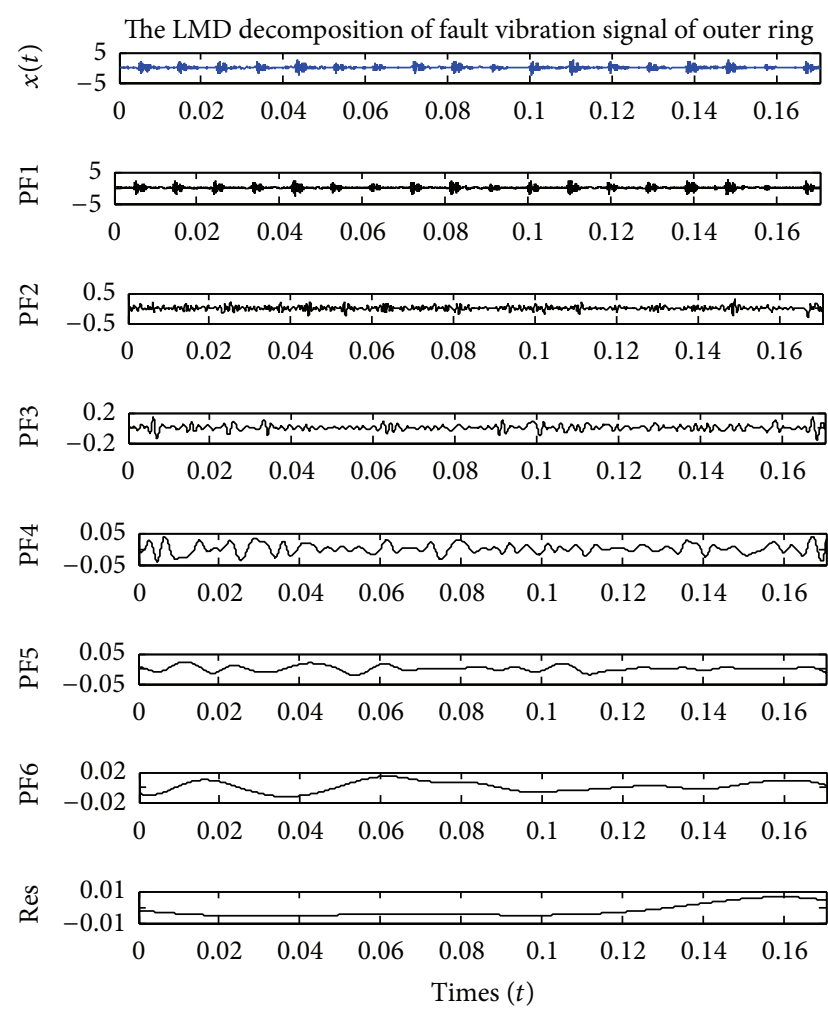

(b) Outer ring fault vibration signal LMD decomposition PF component

FIgURE 8: The time domain and frequency domain waveform of out ring fault vibration signal and the LMD decomposition of the PF component.

at frequency with $140.6 \mathrm{~Hz}$. But the fault characteristic frequency is not obvious enough. It is not unified with the research that rolling element fault is not easy to identify.

\section{The Actual Experimental Analysis}

From the previous sections, use the Case Western Reserve University experimental data of the rolling bearing to verify the effectiveness of the proposed method. To further prove the validity of the proposed method, we generate the practical test data from the rotor experiment platform. The experimental device is shown in Figure 14. The whole device consists of three PCB acceleration sensors, 4-channel dynamic data collecting card (NIUSB-9234), PC, and QPZZ-II rotating machinery vibration analysis and fault experiment system. The outer ring fault of rolling bearing is used in this experiment. The fault bearing parameters are shown in Table 2. The fault characteristic frequency theoretical calculation of rolling bearing is shown as (8).

Combining the theoretical calculation formula of the outer ring fault characteristic frequency and fault bearing parameters, the rolling bearing fault characteristic frequency $f_{0}\left(f_{0}=50.08 \mathrm{~Hz}\right)$ is calculated as follows:

$$
f_{0}=\frac{1}{2} f_{r}\left(1-\frac{d}{D} \cos \alpha\right) Z
$$

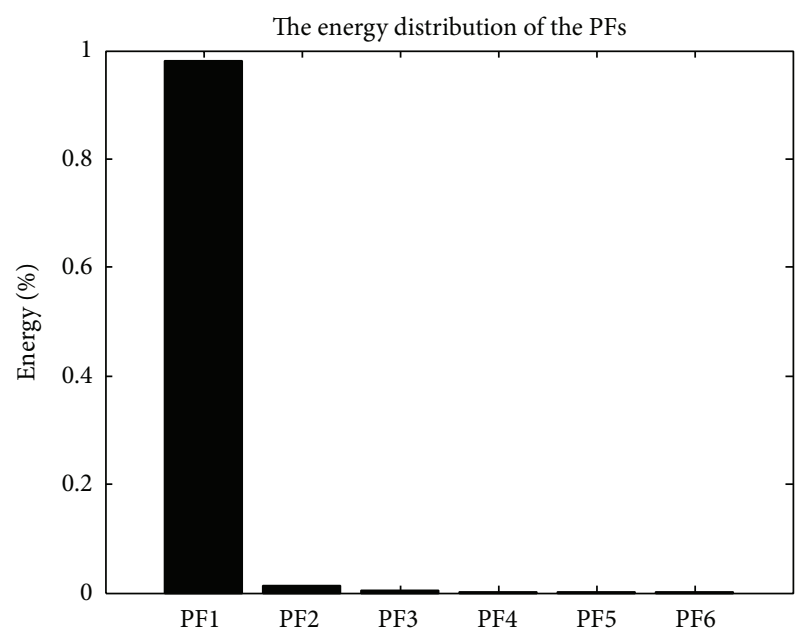

FIgURE 9: The outer fault vibration signal PFs energy distribution.

where $f_{0}$ is outer ring fault characteristic frequency; $f_{r}$ represents rotational frequency $(\mathrm{Hz})$. The rotational frequency of the rolling bearing in this experiment is $620 \mathrm{r} / \mathrm{min}\left(f_{r}=\right.$ $620 / 60 \mathrm{~Hz}=10.33 \mathrm{~Hz}) ; d$ is rolling element diameter $(\mathrm{mm}) ; D$ is bearing pitch diameter $(\mathrm{mm}) ; \alpha$ is contact Angular (contact 


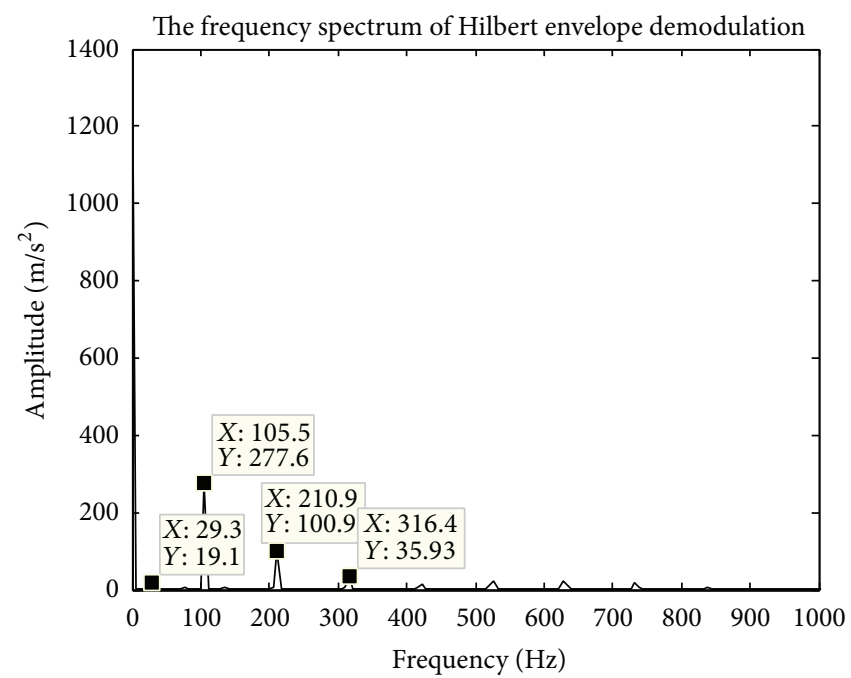

(a) The Hilbert envelope demodulation spectrum

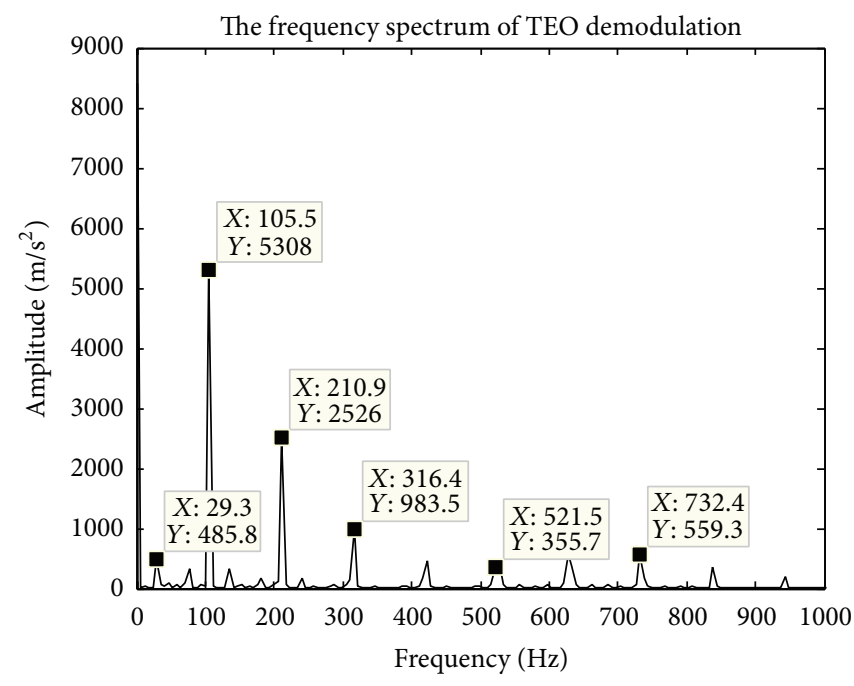

(b) TEO envelope demodulation spectrum

FIGURE 10: The Hilbert of rolling bearing outer ring fault vibration signal and TEO envelope demodulation spectrum.
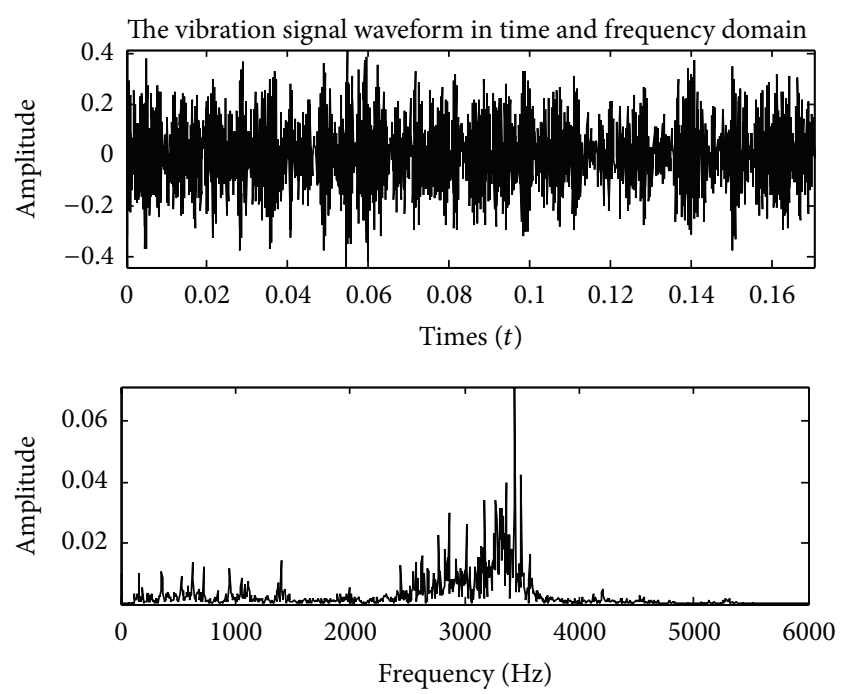

(a) The time domain and frequency domain waveform of rolling fault vibration signal
The LMD decomposition of fault vibration signal of rolling element
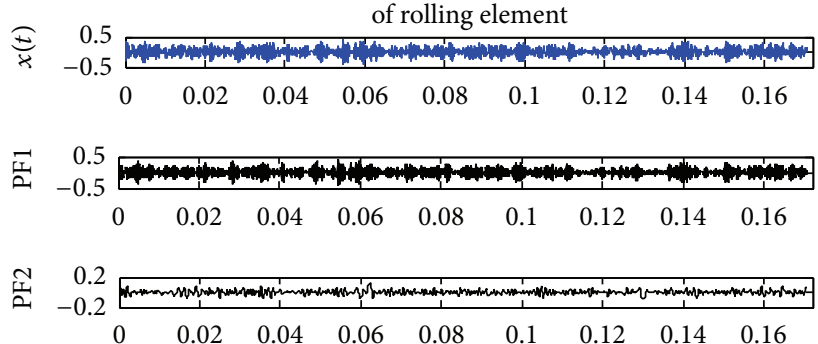

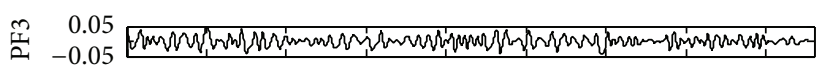
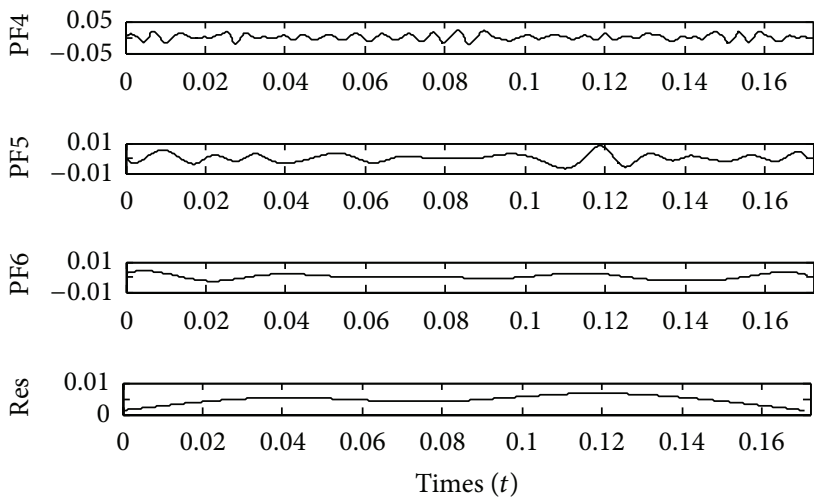

(b) Rolling body fault vibration signal LMD decomposition PF component

FIGURE 11: Time domain and frequency domain waveform of rolling fault vibration signal and the LMD decomposition results.

TABLE 2: The N205EM fault bearing parameter.

\begin{tabular}{lcccc}
\hline Outer ring diameter & Inner ring diameter & Rolling element diameter $(d)$ & Pitch diameter $(D)$ & Rolling element number $(Z)$ \\
\hline $52 \mathrm{~mm}$ & $25 \mathrm{~mm}$ & $7.5 \mathrm{~mm}$ & $39 \mathrm{~mm}$ & 12 \\
\hline
\end{tabular}




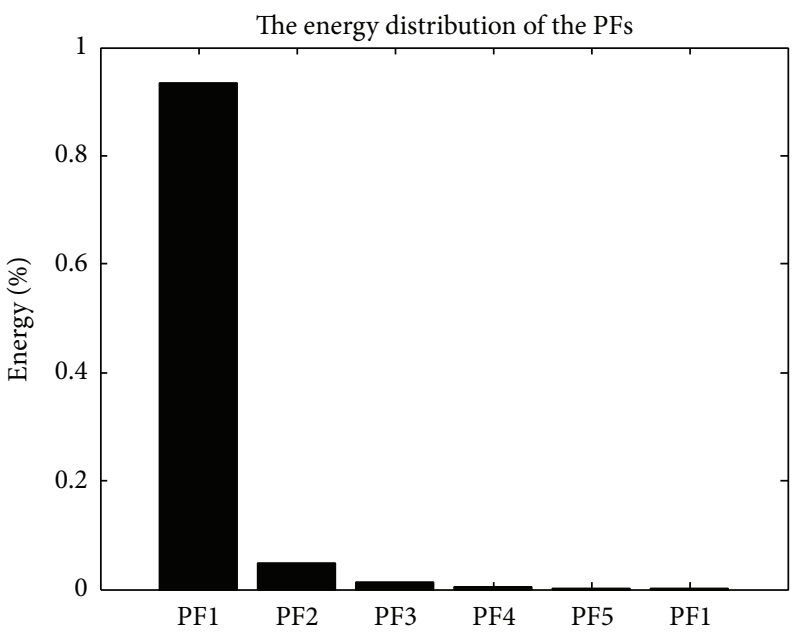

FIGURE 12: Rolling element fault vibration signal energy distribution of PF component.

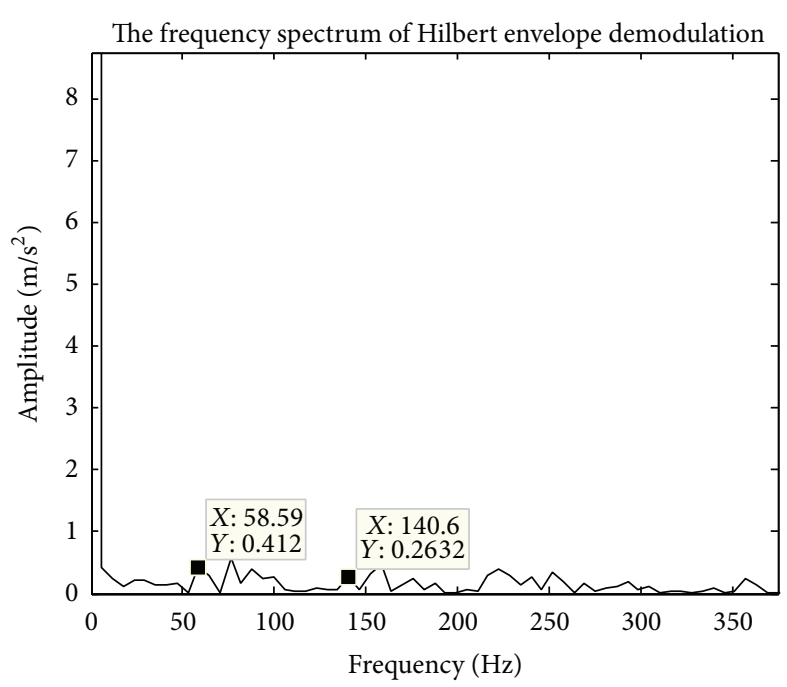

(a) The Hilbert envelope demodulation spectrum

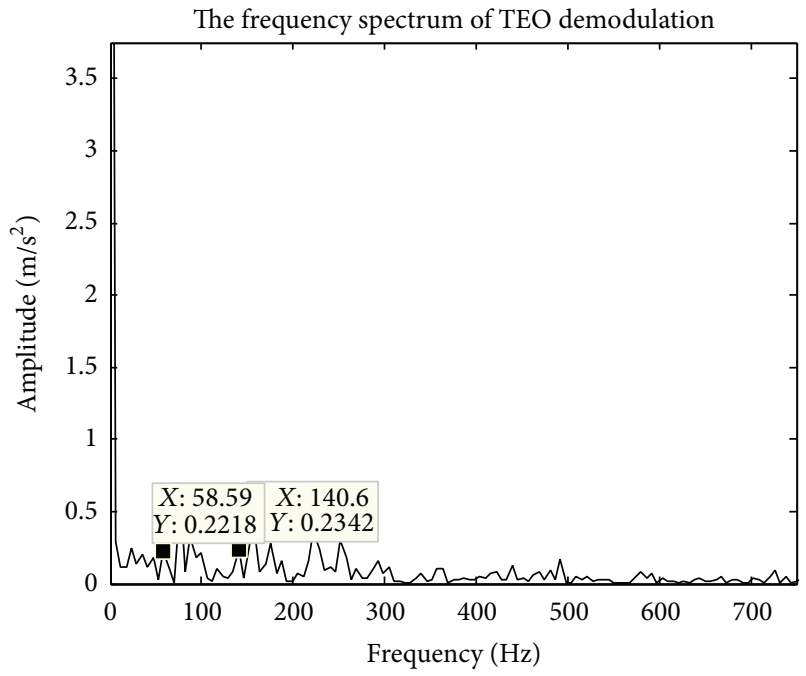

(b) TEO envelope demodulation spectrum

FIGURE 13: The Hilbert of rolling bearing outer ring fault vibration signal and TEO envelope demodulation spectrum.

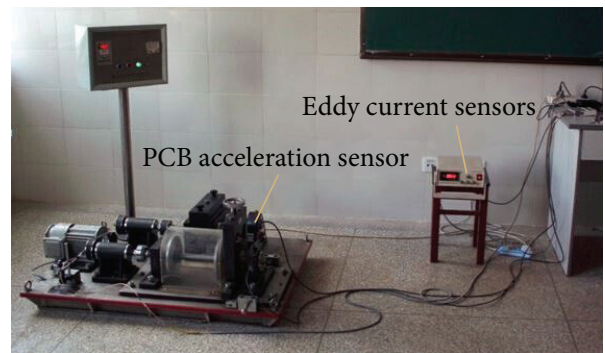

(a) The experimental structure

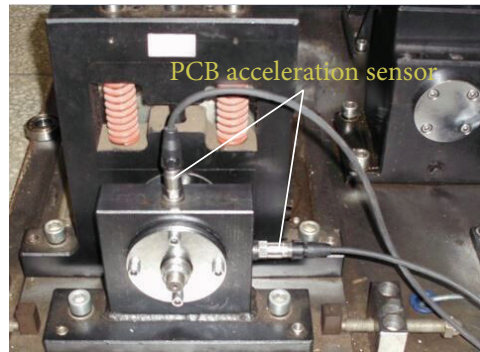

(b) The installation location of the PCB acceleration sensor

FIgURE 14: The experimental facilities. 


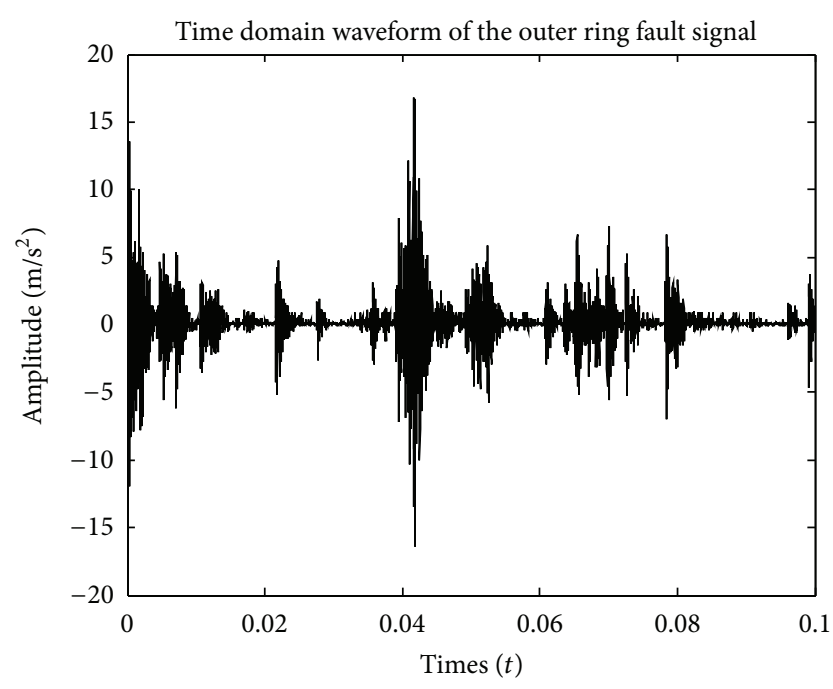

(a) Time domain waveform

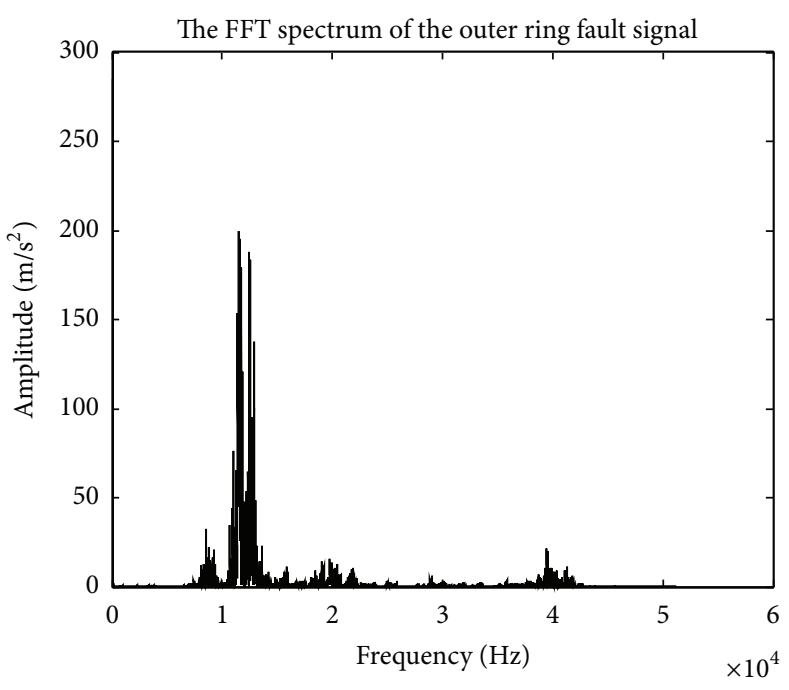

(b) Frequency domain waveform

FIGURE 15: Time and frequency domain waveform of the outer ring fault vibration signal.
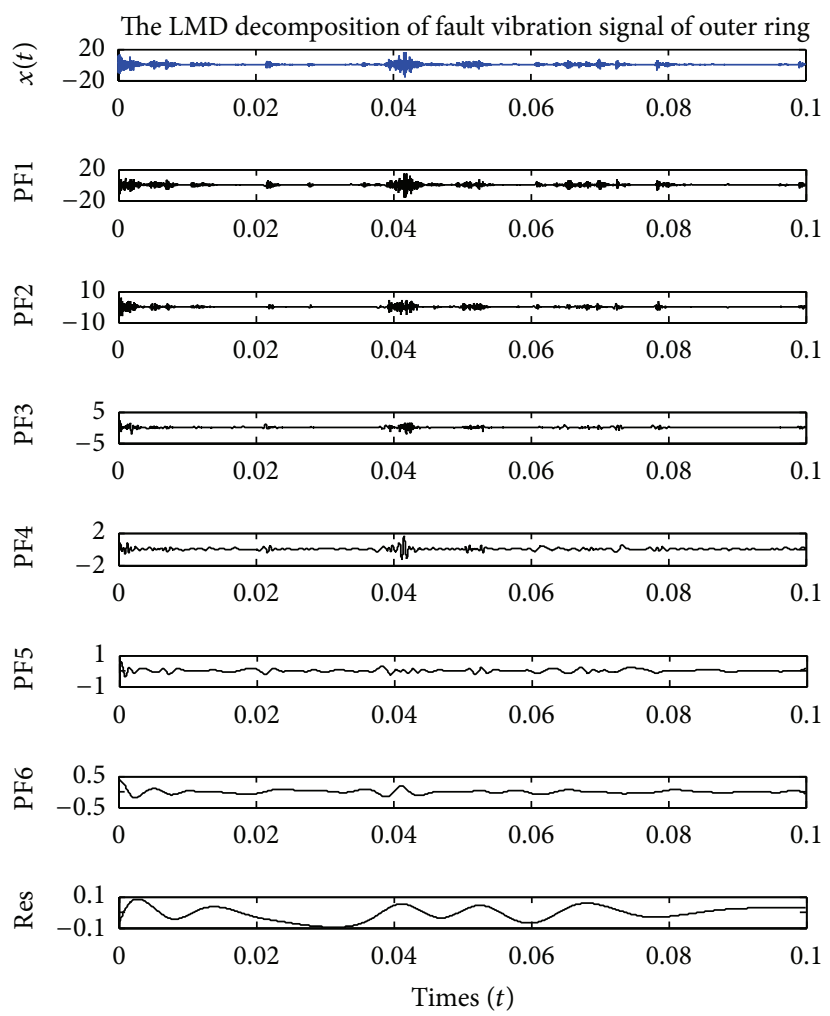

FIGURE 16: PF components of outer ring fault vibration signal LMD decomposition.

angular in this experiment is $\left.0^{\circ}\right) ; Z$ is the number of rolling element.

In this experience, the sample frequency $f_{s}$ of the vibration signals is $102400 \mathrm{~Hz}$ and the data length $N$ is 10240 . The time and frequency domain waveform of the outer ring fault vibration signal is shown in Figure 15. In Figure 15, there exists obvious impact component of the signal in the high frequency part. The main impact components are caused by the fundamental frequency vibration signal of bearing operation in normal, and the bearing fault signal is nonstationary signal. It is difficult to find the fault when directly processing the signal. So it is necessary to extract the impact components of the vibration signal.

In order to extract the fault impact characteristics of vibration signals, decompose the signals by LMD and do envelope demodulation analysis to the effective PFs. The 


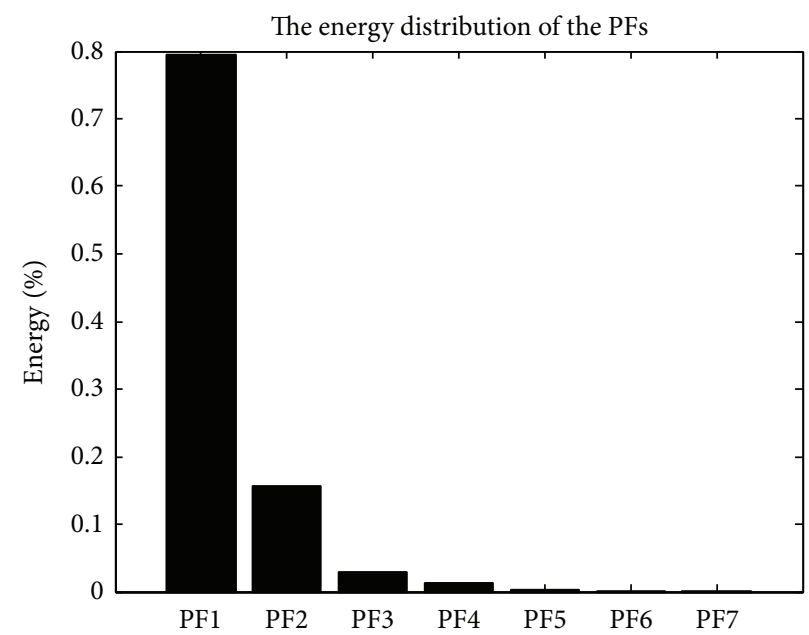

FIGURE 17: The energy distribution of outer ring vibration fault signal.

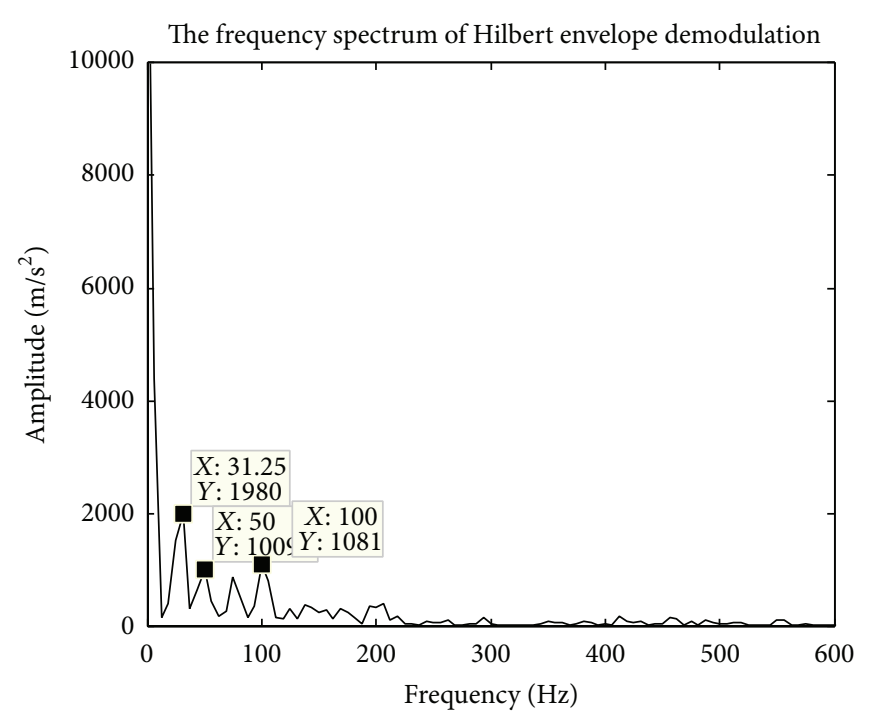

(a) The Hilbert envelope demodulation spectrum

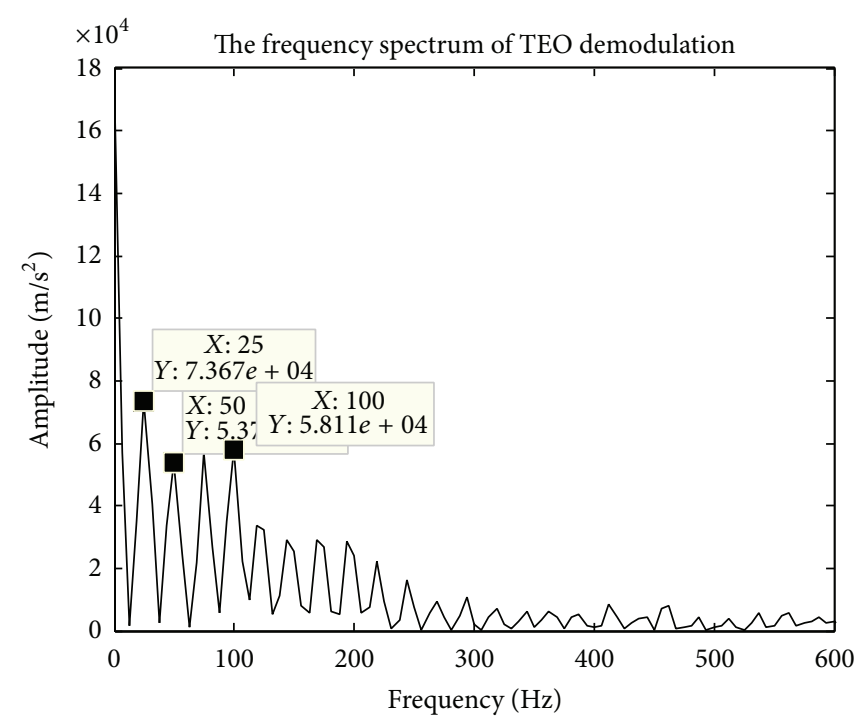

(b) TEO envelope demodulation spectrum

FIGURE 18: The HT and TEO envelope demodulation spectrum of bearing outer ring fault vibration signal.

results of LMD decomposition and energy distribution of PFs are shown in Figure 16 and Figure 17. In Figures 16 and 17, we can see that the energy percentage from component $\mathrm{PF} 3$ to component PF6 in the total energy is small, to be rejected as the pseudocomponent. While the PF1 component contains rich information of the original signal, which is selected for envelope demodulation, the result is shown in Figure 18.

Figure 18 shows the characteristic frequency with $50 \mathrm{~Hz}$, which is similar to the bearing fundamental frequency with $50.08 \mathrm{~Hz}$. At the same time, there also exists the characteristic frequency that is close to its doubling frequency. Because of the effects of outer ring and the environment parameters, there exist certain errors between the theoretical calculation and measured values of rolling fault frequency. Thus we can judge that bearing exhibited outer ring fault at the characteristic frequency with $50.08 \mathrm{~Hz}$. At the same time, from Figure 4 , it evidently can be seen that the obvious crossover frequency with $25 \mathrm{~Hz}$ is obtained by TEO analysis, but in the results of Hilbert analysis, the crossover frequency is at $31.25 \mathrm{~Hz}$. In addition, the amplitude characteristic obtained by TEO demodulation analysis is more outstanding, and it is easy to recognize. The test results are consistent with the previous section analysis.

\section{Conclusions}

Summarizing and analyzing the results mentioned above, the conclusions are obtained as follows.

(1) The vibration signal of rolling bearing is a nonstationary and nonlinear complex signal; it is difficult to extract fault impact component of the rolling bearing and identify its fault type just by analyzing the signal in time-domain or frequency-domain. 
(2) LMD adaptive decomposition method is introduced, and the original vibration signal will be separated into a single pure frequency modulation signal from high frequency to low frequency. Evaluate the energy distribution of each PF component which is obtained by LMD decomposition. Eliminate the influence of residual PF component according to the energy distribution, which provides a basis for the selection of effective PF weight. Then introduce the Hilbert Transform and TEO envelope demodulation to select the effective component of PF envelope demodulation analysis and judge the working condition of rolling bearing according to the Fourier spectrum of PF component.

(3) It can be seen that both of them can identify the operating status of rolling bearing by comparing the results of Hilbert Transform and TEO envelope demodulation. But in the FFT spectrum after TEO demodulation, the fault characteristic frequency amplitude is more outstanding, double frequency and frequency characteristic frequency are more accurate, and the fault recognition is more accurate and more reliable.

The above analysis results show that the envelope demodulation method can effectively and accurately extract the rolling bearing fault feature to diagnose fault. At the same time, it verifies that Teager energy operator demodulation has more advantages than Hilbert Transform demodulation. This method can be widely used in rolling bearing condition and fault diagnosis systems. Due to the limited experimental conditions, the data which is collected in the experiment all belong to a single fault data; all the methods are on the identification for single fault categories. But in the actual operation condition, bearing failure is not always the single failure category, and sometimes it may be composite failure. So the experiment simulates composite fault and collect failure data in actual industry and then use the method mentioned in this paper to identify and diagnose the composite fault of rolling bearings, which will be one of the important works in the study of follow-up work. In addition, deeply analyzing failure mechanism, obtaining the deep reasons for the failure, and providing decision support for the follow-up maintenance of the rotating machinery equipment are also the focus of subsequent research work.

\section{Conflict of Interests}

The authors declare that there is no conflict of interests regarding the publication of this paper.

\section{Acknowledgments}

This work is supported by the National Natural Science Foundation of China (no. 51169007) and Science \& Research Program of Yunnan Province (nos. 2011CI017, 2012CA022, and 2013DH034). Jun Ma also expresses gratitude to Professor Xing Wu in Faculty of Electric Power Engineering of Kunming University of Science and Technology, who provided the experiment platform which Jun Ma has obtained for the test data in Section 5.

\section{References}

[1] P. H. Rodríguez, J. B. Alonso, M. A. Ferrer, and C. M. Travieso, "Application of the Teager-Kaiser energy operator in bearing fault diagnosis," ISA Transactions, vol. 52, no. 2, pp. 278-284, 2013.

[2] P. Tao, H. B. Yang, J. B. Li, H. Y. Jiang, and W. Wei, "Mixeddomain feature extraction approach to rolling bearings faults based on kernel principle component analysis," Journal of Central South University (Science and Technology), vol. 42, no. 6, pp. 3384-3391, 2011.

[3] J. D. Zheng, J. S. Cheng, and Y. Yang, "Generalized empirical mode decomposition and its applications to rolling element bearing fault diagnosis," Mechanical Systems and Signal Processing, vol. 40, no. 1, pp. 136-153, 2013.

[4] L. Gavidia-Ceballos, J. H. L. Hansen, and J. F. Kaiser, "Vocal fold pathology assessment using AM autocorrelation analysis of the Teager energy operator," in Proceedings of the International Conference on Spoken Language Processing (ICSLP'96), pp. 757760, October 1996.

[5] M. Liang and I. Soltani Bozchalooi, "An energy operator approach to joint application of amplitude and frequencydemodulations for bearing fault detection," Mechanical Systems and Signal Processing, vol. 24, no. 5, pp. 1473-1494, 2010.

[6] H. Li, L. Fu, and Y. Zhang, "Bearing faults diagnosis based on teager energy operator demodulation technique," in Proceedings of the International Conference on Measuring Technology and Mechatronics Automation (ICMTMA '09), vol. 1, pp. 594-597, Hunan, China, April 2009.

[7] J.-W. Zhang, N.-H. Zhu, L. Yang, Q. Yao, and Q. Lu, "A fault diagnosis approach for broken rotor bars based on EMD and envelope analysis," Journal of China University of Mining and Technology, vol. 17, no. 2, pp. 205-209, 2007.

[8] W.-C. Tsao, Y.-F. Li, D. D. Le, and M.-C. Pan, "An insight concept to select appropriate IMFs for envelope analysis of bearing fault diagnosis," Measurement, vol. 45, no. 6, pp. 1489-1498, 2012.

[9] M.-C. Pan and W.-C. Tsao, "Using appropriate IMFs for envelope analysis in multiple fault diagnosis of ball bearings," International Journal of Mechanical Sciences, vol. 69, pp. 114-124, 2013.

[10] Z. P. Feng, M. Liang, Y. Zhang, and S. M. Hou, "Fault diagnosis for wind turbine planetary gearboxes via demodulation analysis based on ensemble empirical mode decomposition and energy separation," Renewable Energy, vol. 47, pp. 112-126, 2012.

[11] J. S. Smith, "The local mean decomposition and its application to EEG perception data," Journal of the Royal Society Interface, vol. 2, no. 5, pp. 443-454, 2005.

[12] J.-S. Cheng, K. Zhang, Y. Yang, and D.-J. Yu, "Comparison between the methods of local mean decomposition and empirical mode decomposition," Journal of Vibration and Shock, vol. 28, no. 5, pp. 13-16, 2009.

[13] Y. Yang, J. S. Cheng, and K. Zhang, "An ensemble local means decomposition method and its application to local rub-impact fault diagnosis of the rotor systems," Measurement, vol. 45, no. 3, pp. 561-570, 2012.

[14] J. S. Cheng, Y. Yang, and Y. Yang, "A rotating machinery fault diagnosis method based on local mean decomposition," Digital Signal Processing, vol. 22, no. 2, pp. 356-366, 2012. 
[15] W. Y. Liu, W. H. Zhang, J. G. Han, and G. F. Wang, "A new wind turbine fault diagnosis method based on the local mean decomposition," Renewable Energy, vol. 48, pp. 411-415, 2012.

[16] L. Deng and R. Zhao, "A vibration analysis method based on hybrid techniques and its application to rotating machinery," Measurement, vol. 46, no. 9, pp. 3671-3682, 2013.

[17] M. Feldman, "Hilbert transform in vibration analysis," Mechanical Systems and Signal Processing, vol. 25, no. 3, pp. 735-802, 2011.

[18] J. F. Kaiser, "On a simple algorithm to calculate the energy of a signal," in Proceedings of the International Conference on Acoustics, Speech, and Signal Processing, pp. 381-384, April 1990.

[19] K. A. Loparo, "Bearings vibration data set," Case Western Reserve University, http://csegroups.case.edu/bearingdatacenter/pages/download-data-file.

[20] J. E. Shahan and G. Kamperman, Handbook of Industrial Noise Control, Industrial Press, 1976. 


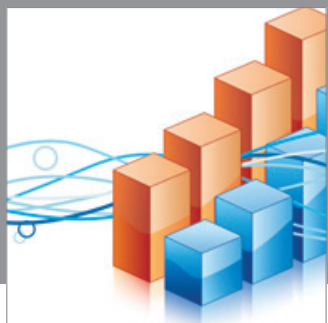

Advances in

Operations Research

mansans

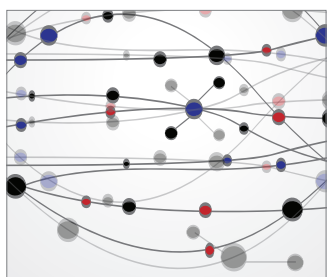

The Scientific World Journal
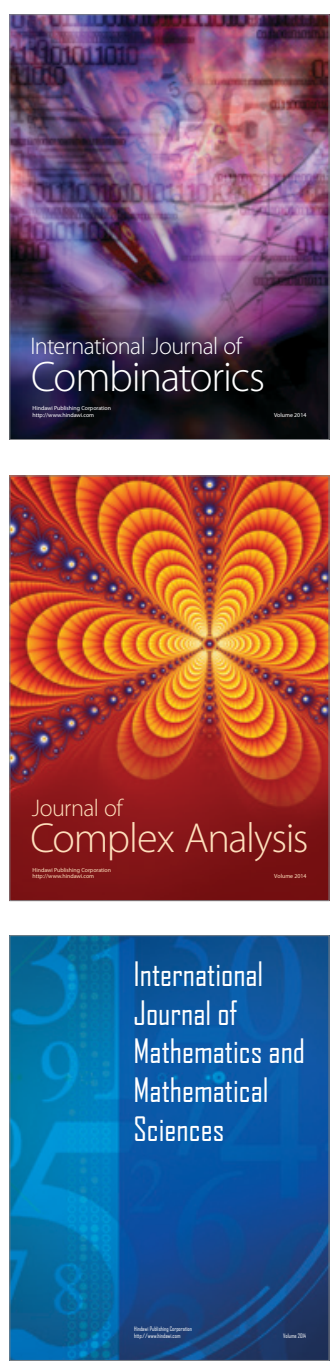
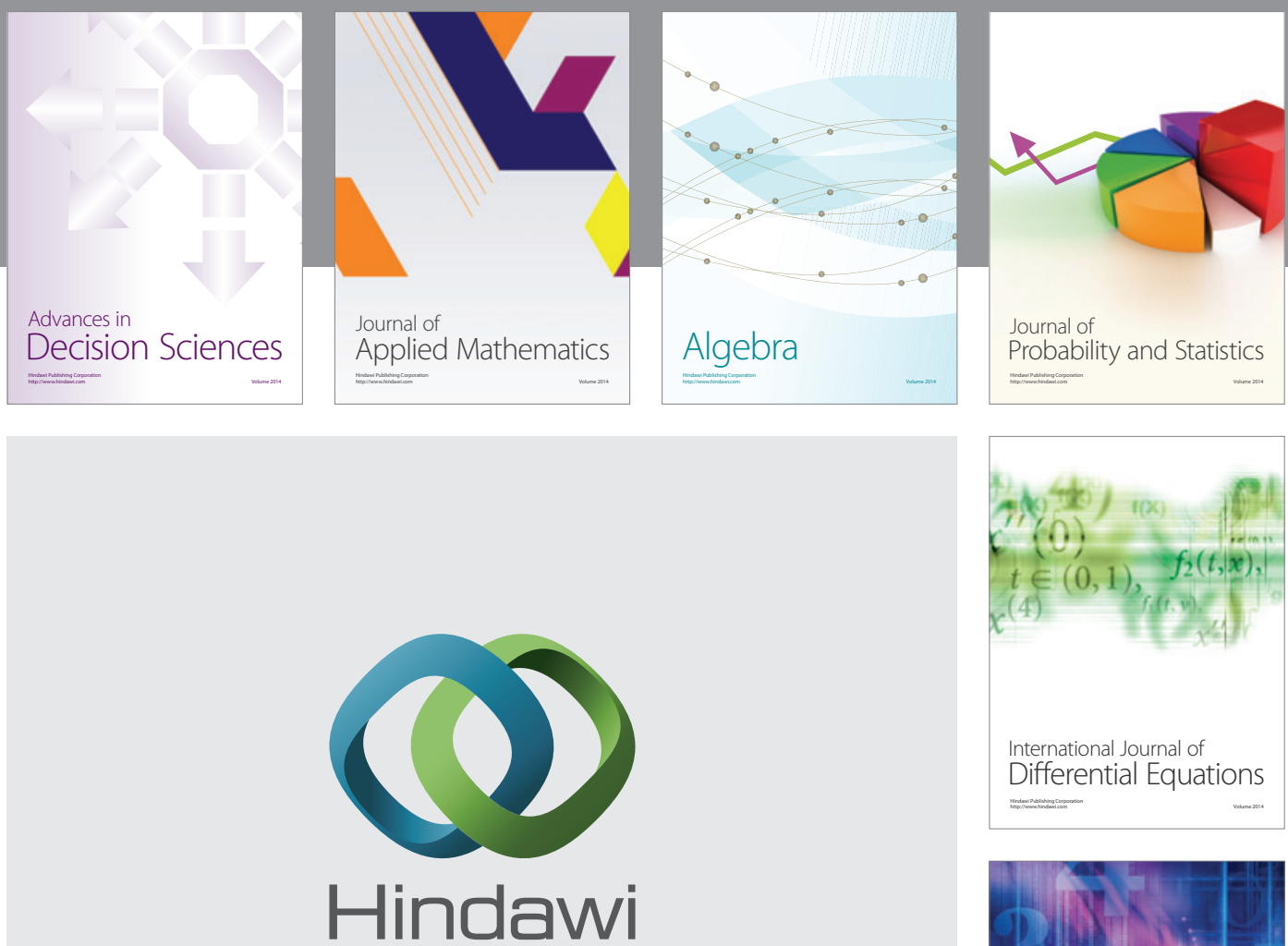

Submit your manuscripts at http://www.hindawi.com
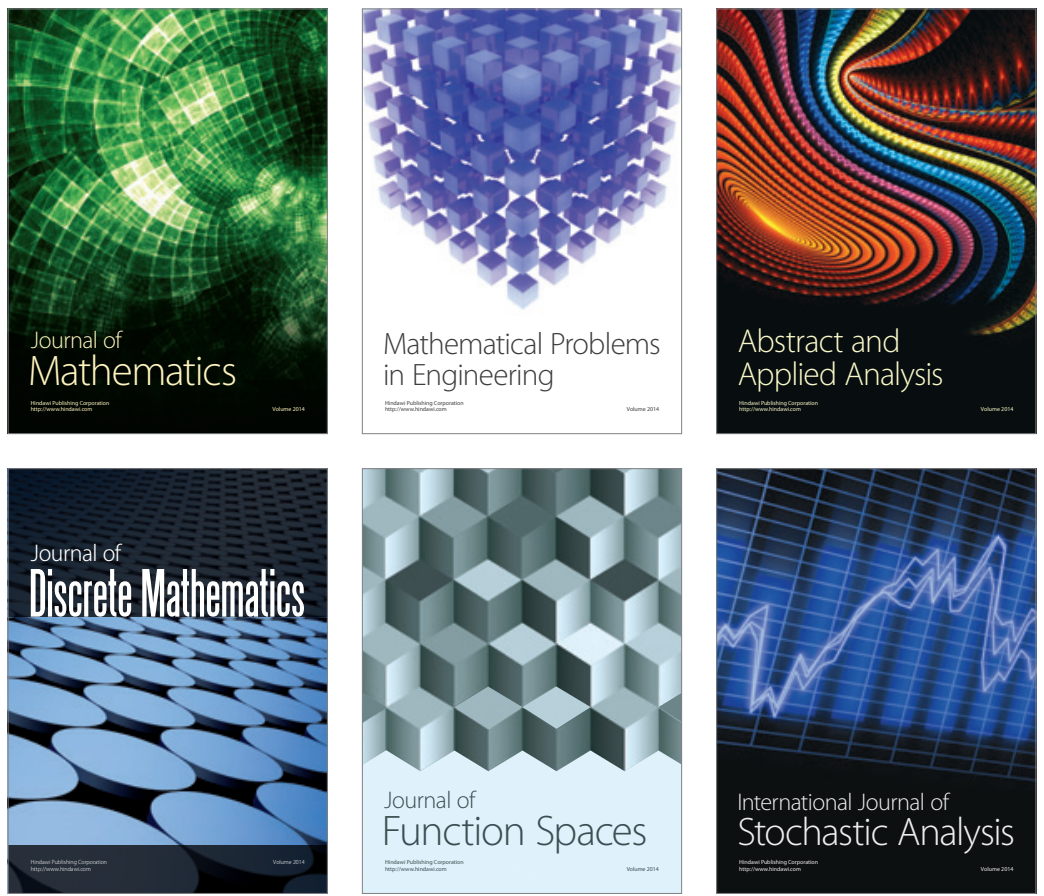

Journal of

Function Spaces

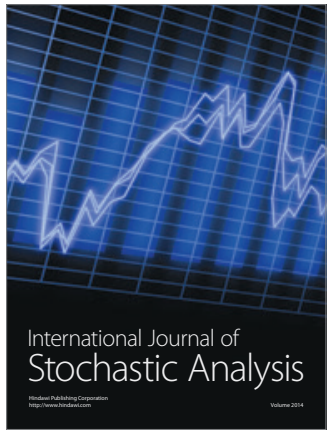

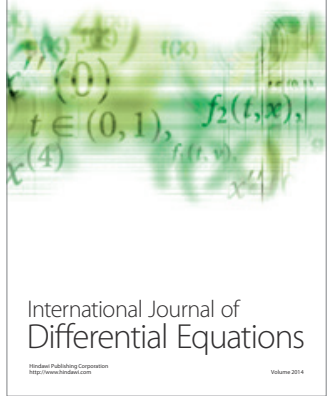
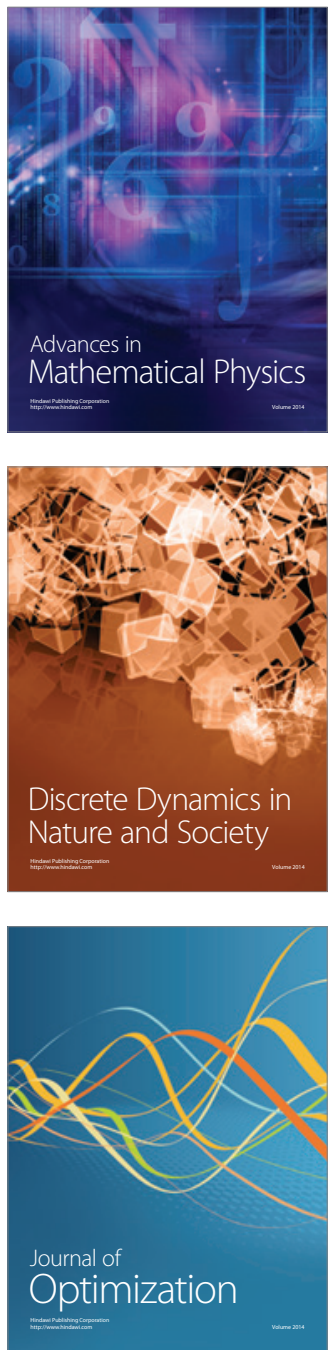